\title{
Alleviation of Neuropathic Pain Hypersensitivity by Inhibiting Neuronal Pentraxin 1 in the Rostral Ventromedial Medulla
}

\author{
Agustin Zapata, ${ }^{1 \star}$ Silvia Pontis, ${ }^{1 \star}$ Raf J. Schepers, ${ }^{1}$ Ruizhong Wang, ${ }^{1}$ Eric Oh, ${ }^{1}$ Alexandra Stein, ${ }^{1}$ Cristina M. Bäckman, ${ }^{2}$ \\ Paul Worley, ${ }^{3}$ Marta Enguita, ${ }^{4}$ M. Alba Abad, ${ }^{4}$ Ramon Trullas, ${ }^{4}$ and Toni S. Shippenberg ${ }^{1}$ \\ ${ }^{1}$ Integrative Neuroscience Branch and ${ }^{2}$ Cellular Neurobiology Branch, National Institute on Drug Abuse, NIH, Baltimore, Maryland 21224, ${ }^{3}$ Department of \\ Neuroscience, Johns Hopkins University School of Medicine, Baltimore, Maryland 21205, and ${ }^{4}$ Neurobiology Unit, Institut d'Investigacions Biomèdiques \\ de Barcelona (CSIC/IDIBAPS) and Centro de Investigación Biomédica en Red sobre Enfermedades Neurodegenerativas (CIBERNED), 08036 Barcelona, \\ Spain
}

Peripheral nerve injury causes spontaneous and long-lasting pain, hyperalgesia, and allodynia. Excitatory amino acid receptordependent increases in descending facilitatory drive from the brainstem rostral ventromedial medulla (RVM) contribute to injuryevoked hypersensitivity. Although increased excitability likely reflects changes in synaptic efficacy, the cellular mechanisms underlying injury-induced synaptic plasticity are poorly understood. Neuronal pentraxin 1 (NP1), a protein with exclusive CNS expression, is implicated in synaptogenesis and AMPA receptor recruitment to immature synapses. Its role in the adult brain and in descending pain facilitation is unknown. Here, we use the spared nerve injury (SNI) model in rodents to examine this issue. We show that SNI increases RVM NP1 expression and constitutive deletion or silencing NP1 in the RVM, before or after SNI, attenuates allodynia and hyperalgesia in rats. Selective rescue of RVM NP1 expression restores behavioral hypersensitivity of knock-out mice, demonstrating a key role of RVM NP1 in the pathogenesis of neuropathic pain.

\section{Introduction}

Peripheral nerve injury produces hypersensitivity to both noxious and non-noxious stimuli. Maladaptive plasticity within brainstem descending pathways linking the rostral ventromedial medulla (RVM) to the spinal cord is implicated in the pathogenesis of neuropathic pain. Persistent pain after injury is linked to enhanced activation of descending modulatory circuits and increased net descending facilitatory drive, which leads to an amplification and spread of pain (Urban and Gebhart, 1999; Vanegas and Schaible, 2004). Blockade of excitatory amino acid receptors in the RVM attenuates behavioral hypersensitivity and the enhanced responsiveness of spinal cord neurons to noxious and innocuous stimuli, suggesting the involvement of excitatory amino acid transmission in the descending facilitation of neuropathic pain (Sanoja et al., 2008). Although increased RVM excitability likely reflects altered synaptic efficacy, the mechanisms

\footnotetext{
Received June 6, 2012; revised July 9, 2012; accepted July 10, 2012

Author contributions: A.Z., S.P., R.J.S., P.W., R.T., and T.S.S. designed research; A.Z., S.P., R.J.S., R.W., E.O., A.S., C.M.B., M.E., M.A.A., and R.T. performed research; A.Z., S.P., R.J.S., R.W., and T.S.S. analyzed data; A.Z., R.T., and T.S.S. wrote the paper.

These studies were supported by the NIDA Intramural Research Program and by Grants SAF2008-03514 and SAF2011-23550 from Ministerio de Economia y Competitividad of Spain (to R.T.) and Grant NS39156 (to P.W.).

*A.Z. and S.P. contributed equally to this work.

The authors declare no competing financial interests.

Correspondence should be addressed to Dr. Agustin Zapata, Integrative Neuroscience Branch, NIDA IRP, 333 Cassell Drive, Baltimore, MD 21224. E-mail: Azapata@mail.nih.gov.

DOI:10.1523/JNEUROSCI.2730-12.2012

Copyright $\odot 2012$ the authors $\quad 0270-6474 / 12 / 3212431-06 \$ 15.00 / 0$
}

underlying injury-induced synaptic plasticity in the pain modulatory circuitry remain ill defined.

An early event in excitatory synapse formation is the clustering of postsynaptic glutamate (Glu) receptors to the synapse (O'Brien et al., 1997). Targeting and cell surface clustering of these receptors to the synapse are essential for efficient excitatory synaptic transmission (O'Brien et al., 1998). Among the molecules hypothesized to act as early mediators of synaptic AMPA receptor recruitment are the neuronal pentraxins (NPs), proteins exclusively expressed in the CNS that are homologous to the C-reactive and acute-phase proteins in the immune system (Tsui et al., 1996). NP1, a member of the NP family (Xu et al., 2003; Sia et al., 2007; Hossain, 2008), physically associates with fast excitatory GluR1 and GluR4 AMPA receptor subunits in hippocampal cultures and artificial synapses where it acts trans-synaptically to cluster AMPA receptors at postsynaptic sites and promote excitatory synaptogenesis (O'Brien et al., 1999; Xu et al., 2003; Sia et al., 2007). Little, however, is known regarding the function of NP1 in the adult brain in vivo. Given that excitatory amino aciddependent increases in descending facilitatory drive from the RVM are critical for the development of neuropathic pain and that recombinant NP1 promotes synaptic AMPA receptor clustering, we used the spared nerve injury model (SNI) of neuropathic pain to examine whether NP1 gene deletion or silencing of NP1 expression in the RVM protects against allodynia and hyperalgesia produced by peripheral nerve injury. Our studies reveal that NP1 is selectively induced in the RVM following SNI and that gene knockout or the specific silencing of NP1 in the RVM, 
either before or after injury, attenuates SNI-evoked behavioral hypersensitivity. Furthermore, selective rescue of NP1 expression in the RVM restores the allodynia and hyperalgesia that is attenuated in NP1 knock-out (KO) mice.

\section{Materials and Methods}

Subjects. Male Sprague Dawley rats (Charles River; 300-450 g), NP1 KO mice (Kirkpatrick et al., 2000), and their wild-type littermates (C57BL/6 background; obtained from heterozygous crossings: F6-8) were maintained in temperature- and humidity-controlled facilities accredited by the American Association for the Accreditation of Laboratory Animal Care. Food and water were available ad libitum. Experiments were approved by the NIDA IRP Institutional Care and Use Committee and conducted in accordance with The Ethical Guidelines for Investigation of Experimental Pain in Conscious Animals (International Association for the Study of Pain) (Zimmermann, 1983).

Lentiviral vectors. The self-inactivating bicistronic lentiviral transfer vector constructs pWPI and PLVTHM and the second-generation lentivirus psPAX2 packaging and $\mathrm{pMD} 2 \mathrm{G}$ envelope plasmids were obtained from addgene (http://www.addgene.org/). The pWPI-NP1 lentiviral vector that drives NP1 overexpression and the pLVTHM-NP1-short hairpin RNA interference (shRNAi) vector that carries the H1 RNA polymerase III promoter to enable expression of a shRNA for NP1 knockdown were obtained as previously described (Abad et al., 2006). We used the pLVTHM lentiviral vector carrying a silencing cassette expressing a shRNAi against no known target (Random-shRNAi) as a control for the NP1-shRNAi vector. The sequence of the NP1-shRNAi vector is as follows: sense, $5^{\prime}$-gatccccGTACAG CCGCCTCAATTCTttcaagagaAGAATTGAGGCGGCTGTACttttt-3'; and antisense, $5^{\prime}$-agctaaaaaGTACAGCCGCCTCAATTCTtctcttgaaAGAA TTGAGGCGGCTGTACggg- $3^{\prime}$. The sequence in capitals is the NP1 target sequence that corresponds to bases 1004-1022 of NP1 mRNA (GenBank Accession number U18772). The sequence of the RandomshRNAi is as follows: sense, $5^{\prime}$-gatccccGCAGTGCAATATCGGAAACtt caagagaGTTTCCGATATTGCACTGCttttt- ${ }^{\prime}$; and antisense, $5^{\prime}$-agctaaaa aGCAGTGCAATATCGGAAACtctcttgaaGTTTCCGATATTGCACTGC ggg- 3 '. The efficiency of the NP1-shRNAi silencing cassette to knock down NP1 expression was tested by immunoblotting in SH-SY5Y cell clones that permanently overexpress NP1 using actin for normalization.

The viral particles were produced by transient transfection of $293 \mathrm{~T}$ cells. High-titer stocks $\left[7 \times 10^{6}\right.$ transduction units (TU) per microliter] were obtained by ultracentrifugation and resuspension of the viral pellet in TNE buffer (50 mm Tris- $\mathrm{HCl}, \mathrm{pH} 7.5,130 \mathrm{~mm} \mathrm{NaCl}$, and 1 mm EDTA). The infectious viral titer was determined by flow cytometry with a FACScalibur (Beckton-Dickinson) measuring 293T cells expressing GFP $48 \mathrm{~h}$ after transduction with the lentiviral vectors. Viral stocks were stored at $-20^{\circ} \mathrm{C}$ and used within 1 month.

Intra-RVM lentivirus infusions. The RVM is loosely defined as the midline pontomedullary region including the nucleus raphe magnus and the adjacent reticular formation (Fields and Heinricher, 1985). Guide cannulae (C315, Plastics One) were stereotaxically implanted $1 \mathrm{~mm}$ dorsal to the RVM. The coordinates were as follows (in $\mathrm{mm}$ ): rats: anteroposterior (AP), -10.5 to -11.0 ; lateral (L), -0.0 ; ventral (V), -9.7; and mice: AP, $-6.1, \mathrm{~L},-0.0 ; \mathrm{V},-5.0$. Animals were single housed after surgery for the remainder of the study. After recovery from surgery (4-5 d), animals were lightly anesthetized with isoflurane and the microinjection needle, which extended $1 \mathrm{~mm}$ below the guide cannula tip, was inserted. One microliter of the lentiviral solution $\left(\sim 7 \times 10^{6} \mathrm{TU}\right)$ was infused over $1 \mathrm{~min}$. The injector was left in place for $4 \mathrm{~min}$ to allow for diffusion. Experiments started 7-10 d after infusion, except for experiments assessing the effects of silencing after SNI, which commenced 3-4 $\mathrm{d}$ after infusion. In vitro data showed that the lentivirus vector rapidly integrates into the cell genome and significantly reduces NP1 protein levels within $48 \mathrm{~h}$.

Spared nerve injury. The left hindlimb was immobilized under general anesthesia (rats, Equitesin; mice, ketamine-xylazine), and an incision made through the biceps femoralis muscle. The sciatic nerve was exposed and the common peroneal and tibial branches were ligated and transected, and a 1-2 mm section was removed with care to avoid sural nerve damage (Decosterd and Woolf, 2000; Bourquin et al., 2006). After transection, the muscle fascia and skin were sutured. Subsequent nociceptive testing (von Frey test and pinprick) was conducted on the sural skin territory of the plantar surface.

Assessment of nociceptive thresholds. Mechanical thresholds were determined with the von Frey method (Levin et al., 1978) using 0.6-26.0 g monofilaments (Stoelting) for rats, and 0.04-6.0 g filaments for mice, as described previously (Schepers et al., 2008). The up-and-down method (Chaplan et al., 1994) was used for computation of the 50\% response threshold. For the pinprick test (Tal and Bennett, 1994), a 30 gauge needle was gently applied to the plantar surface of the hindpaw without breaking the skin. Nocifensive reactions were measured as the paw lift duration (in seconds). Testing was conducted by an experimenter blinded to the treatment condition.

$R T-P C R$. RVM tissue was dissected and immediately processed for RNA isolation and DNase I treatment (RNAqueous-Micro Ambion kit, Applied Biosystems) following manufacturer instructions. For cDNA synthesis, total RNA was mixed using the Superscript III reverse transcriptase kit (Invitrogen). RT-PCR was performed as duplicate determinations with specific Taqman probes from the rat Probe Library (Exiqon A/S) designed for primers to rat NP1 (forward, $5^{\prime}$-cct tga cct ccc tac acc ag- $3^{\prime}$; reverse, $5^{\prime}$ ctc cgg gtc gat tgt ctt t-3'), housekeeping genes Ribosomal protein S18 (forward, 5' -cgagtactcaacaccaacatcg-3'; reverse, $5^{\prime}$-caccacat gagcgtatctcc- $3^{\prime}$ ), and hydroxymethylbilane synthase (forward, $5^{\prime}$ tcc ctg aag gat gtg cct ac $3^{\prime}$; reverse, $5^{\prime}$ aca agg gtt ttc ccg ttt g $3^{\prime}$ ). NP1 normalization to both endogenous control genes led to similar results.

Immunohistochemistry. Two or 14 days after SNI, rats were deeply anesthetized with Equitesin $(3 \mathrm{ml} / \mathrm{kg})$ and transcardially perfused with 50 $\mathrm{ml}$ of saline solution followed by $150 \mathrm{ml}$ of $4 \%$ paraformaldehyde. Tissue was postfixed in paraformaldehyde $4 \%$ for $1 \mathrm{~h}$ and then stored in $30 \%$ sucrose for $3 \mathrm{~d}$. Forty micrometer sections were collected $\geq \mathrm{RVM}$ : AP -10.04 to $-11.30 \mathrm{~mm}$; Periaqueductal Gray (PAG): AP -4.80 to -8.30 $\mathrm{mm}$; spinal cord L4-L6] and processed for immunohistochemistry. Dual immunostaining of NP1 and tryptophan hydroxylase (TPH) was performed by sequentially incubating sections with NP1 primary antibody (BD Bioscience; dilution 1:1000) and Alexa Fluor 568 (Invitrogen; dilution 1:1000) followed by TPH primary antibody (Santa Cruz Biotechnology; dilution 1:500) and Alexa Fluor 488 (Invitrogen; dilution 1:1000). Dual immunostaining of NP1 and glutamic acid decarboxylase-67 (GAD-67) (primary antibody from Santa Cruz Biotechnology; dilution 1:500) was performed in alternate sections according to the same protocol. A total of three sections per animal, representing one of every fifth section were analyzed. Total number of NP1-positive cells, percentage of TPH-positive cell expressing NP1, and percentage of GAD-67-positive cells expressing NP1 were evaluated independently by two observers blind to the treatment. Single labeling of NP1 was performed in ipsilateral spinal cord and dorsal root ganglia (DRGs) sections, and the number of cells expressing NP1 was quantified.

\section{Results}

Immunohistochemistry revealed low NP1 expression in the spinal cord dorsal horn (SpC), RVM, and PAG of control rats and undetectable levels in DRGs. SNI induced a sustained increase in NP1 immunoreactivity in the RVM (Fig. 1a). NP1 immunoreactivity in sham-operated animals was observed in a subset (30$40 \%$ ) of GAD67- and TPH-containing neurons (Fig. $1 b$ ) and was primarily restricted to the nuclear compartment. Following SNI, NP1 was localized to puncta distributed throughout the cytoplasm and the number of GABAergic, but not TPH, neurons expressing NP1 significantly increased (Fig. 1b). This increase was selective; NP1 expression in DRG, spinal cord, and PAG was unaltered (Fig. 1a).

We tested whether lentiviral-mediated silencing of NP1 in the RVM (Fig. 2a-e) affects mechanical hypersensitivity that develops following SNI. Silencing NP1 before nerve injury attenuated the development of mechanical allodynia and hyperalgesia (Fig. $2 f$ ). Moreover, when silencing was conducted after injury, the 

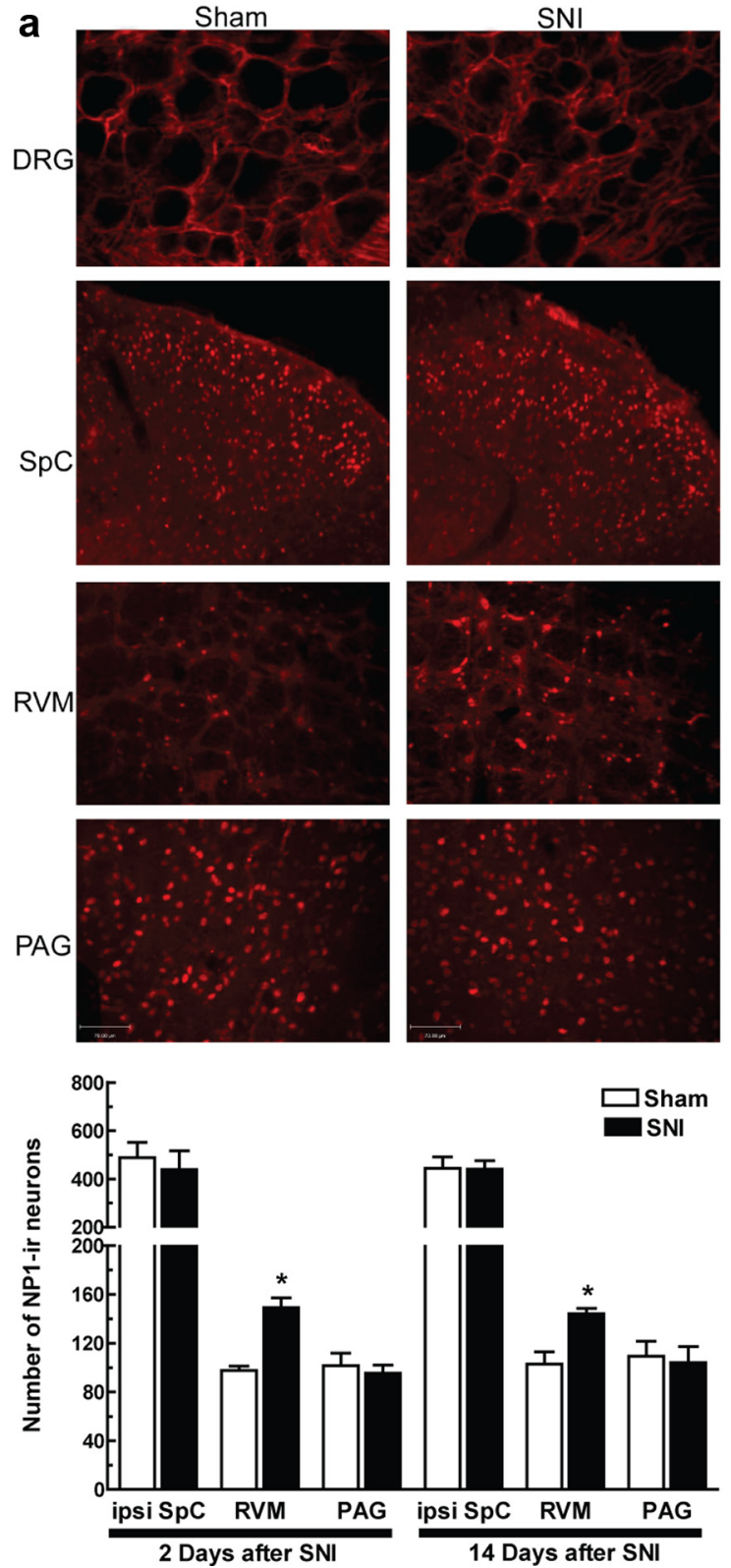

b

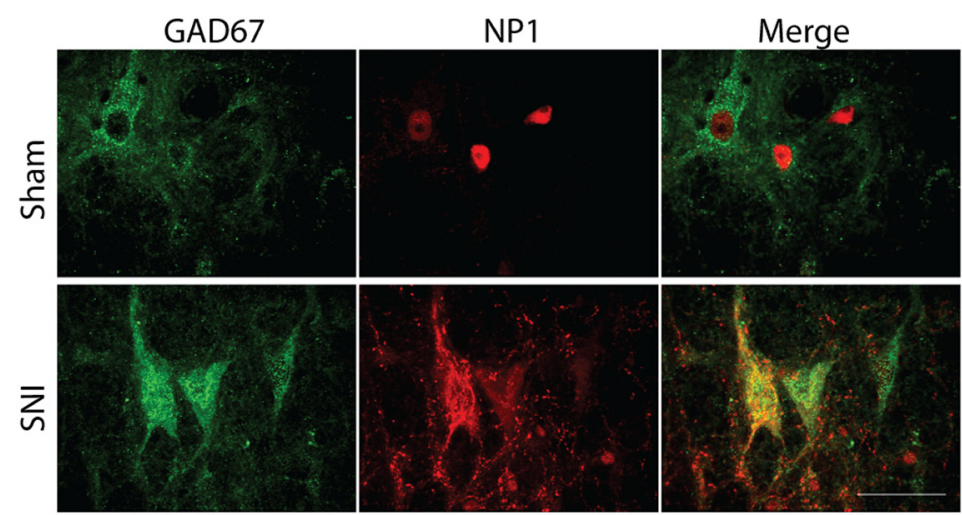

$\mathrm{TPH}$
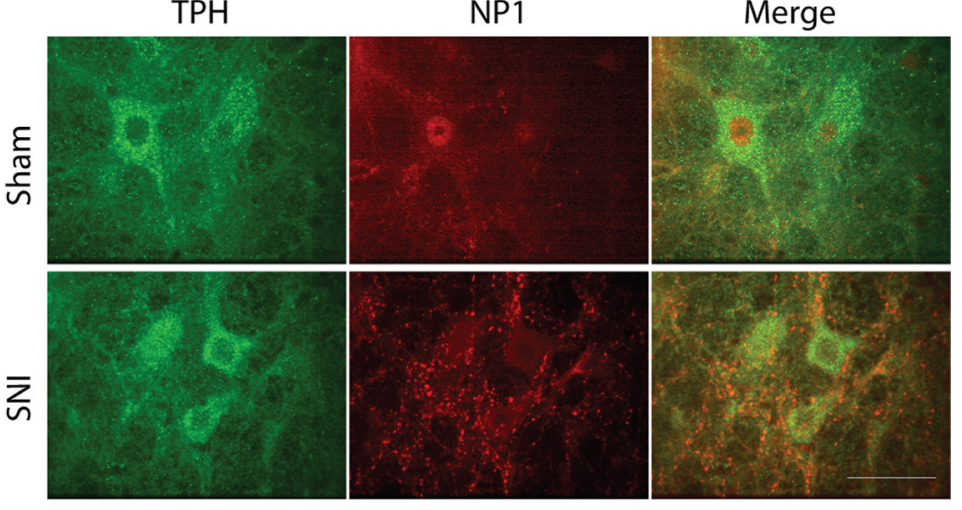

GAD67 cells positive for NP1

TPH cells positive for NP1
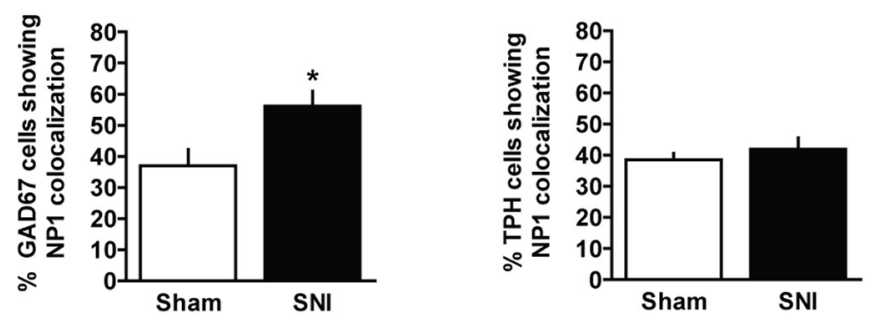

Figure 1. SNI selectively induces NP1 in the RVM and induction is restricted to a subset of GABAergic neurons. $a, N P 1$ immunoreactivity is increased in the RVM, but not in the DRG, SpC, or PAG, $2 \mathrm{~d}$ after SNI (representative picture and histogram, $t=-5.83, \mathrm{df}=10,{ }^{*} p=0.0002, t$ test). NP1 induction persists for at least 2 weeks after $\mathrm{SNI}\left(\right.$ histogram, $t=-3.83, \mathrm{df}=10,{ }^{*} p=0.003$, $t$ test). $\boldsymbol{b}$, RVM sections obtained $2 \mathrm{~d}$ after SNI or sham surgery were stained for NP1 and either TPH or GAD67 (markers of 5 -HT and GABAergic cells, respectively). Nerve injury increased the number of GAD67 cells immunoreactive for NP1 ( $t=2.67, \mathrm{df}=9,{ }^{*} p=0.03, t$ test), whereas the number of cells coexpressing TPH and NP1 was unaltered.

expression of hypersensitivity was attenuated (Fig. $2 g$ ). NP1 silencing did not alter basal nociceptive thresholds in uninjured animals (Fig. $2 g$ ), suggesting the specific involvement of NP1 in the pathogenesis of persistent pain resulting from nerve injury. The effects of the silencing vector were attenuated by shifting the infusion site $1 \mathrm{~mm}$ dorsal to the RVM. Mechanical thresholds (mean \pm SEM) $48 \mathrm{~h}$ after infusion of the silencing lentiviral vector were $8.5 \pm 1.9 \mathrm{~g}$ (dorsal to target) and $2.5 \pm 0.3 \mathrm{~g}$ (at target); baseline thresholds were 11.6 and $10.2 \mathrm{~g}$, respectively. These data suggest a specific role for NP1 within the RVM in the antiallodynic and analgesic effects of NP1 silencing.

Consistent with our studies in rats, SNI-induced mechanical allodynia was reduced in NP1 knock-out mice (Fig. $2 h$, left). The phenotype of knock-out mice was reversed by specifically rescuing NP1 expression in the RVM (Fig. $2 h$, right).

\section{Discussion}

We found increased levels of NP1 in the RVM, but not other painrelated CNS areas, in a rodent model of chronic neuropathic pain. This increase appeared to be accompanied by subcellular redistribution and to occur in GABAergic, but not 5-HT, neurons. Inhibition of NP1 expression in the RVM, or deletion of the NP1 gene resulted in alleviation of nerve injury associated hyperalgesia and allodynia. Furthermore, restoring NP1 expression selectively in the RVM is sufficient to reinstate the pain-related behavioral phenotype. Together, these data indicate that peripheral nerve injury induces NP1 in the RVM and that increased expression therein is essential for the development and expression of nerve injury-evoked pain.

Previous studies have indicated a role for NP1 in pathological neurodegenerative states, including apoptotic cell death in pri- 
a

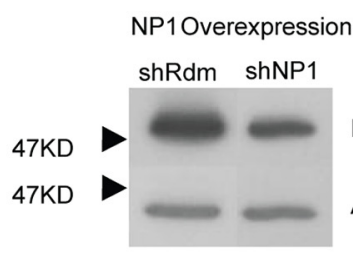

b

C

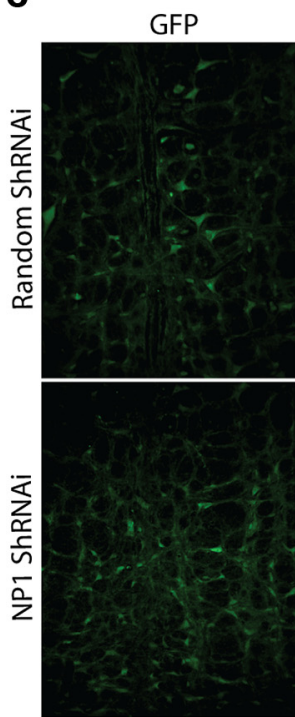

d

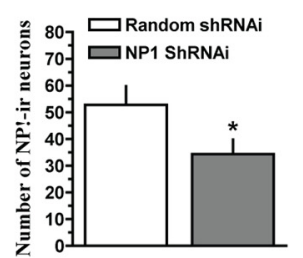

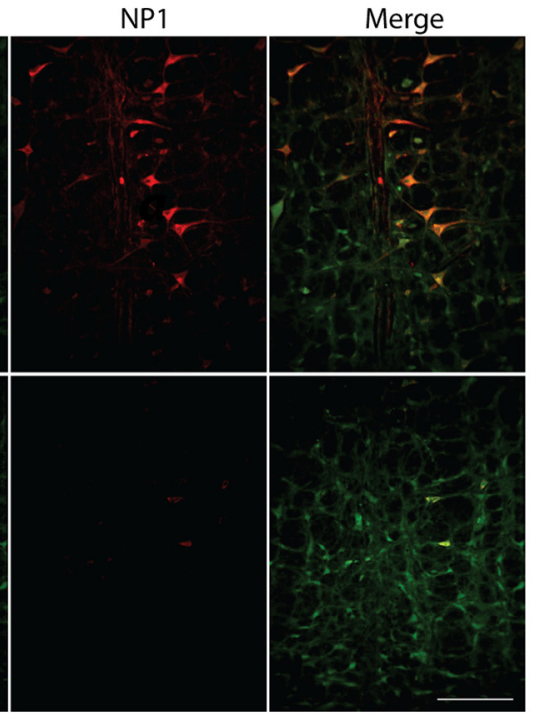
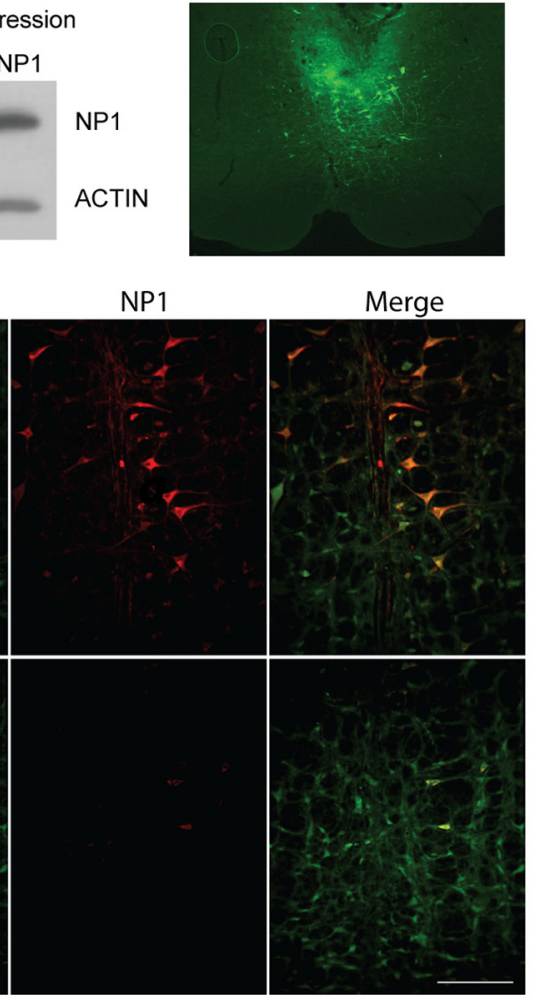

Merge

e

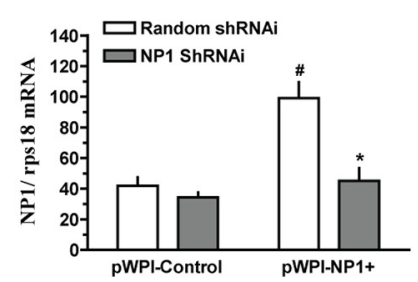

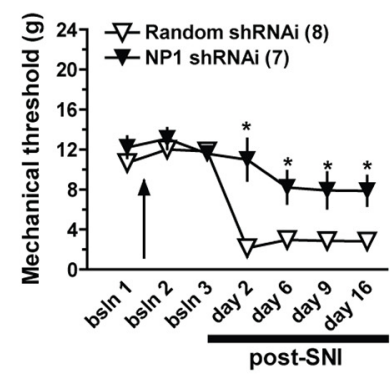

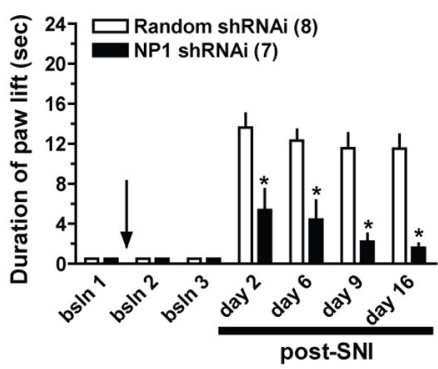

g
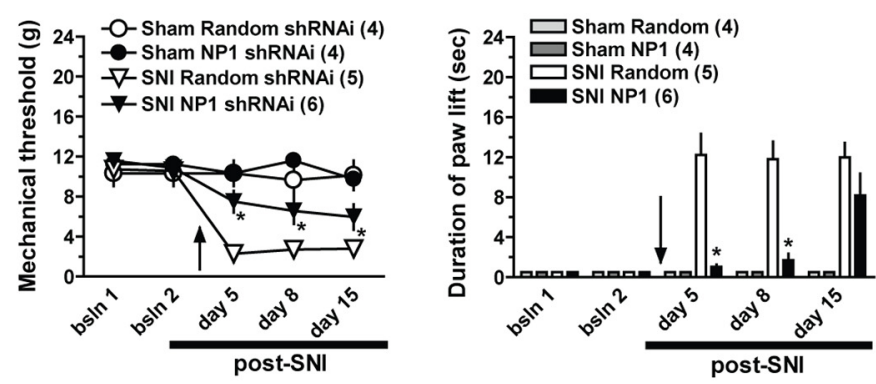

h

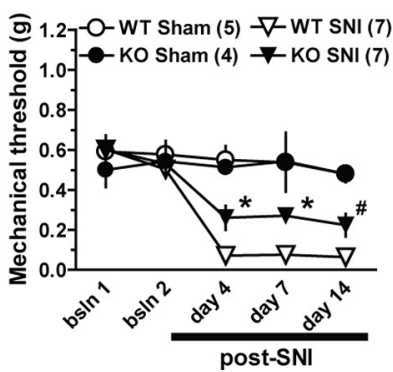

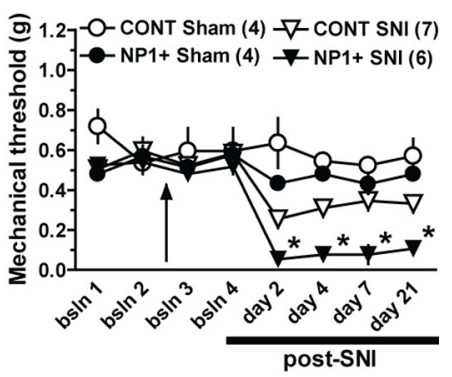

Figure 2. NP1 in the RVM is involved in SNI-evoked mechanical allodynia and hyperalgesia. $\boldsymbol{a}$, The efficacy of a shRNAi sequence in silencing NP1 expression was confirmed by immunoblotting in SH-SY5Y neuroblastoma cells constitutively overexpressing NP1. The NP1 shRNAi cassette (or a random shRNAi for control vectors) was then cloned into the lentiviral pLVTHM vector (which included a reporter GFP cassette) for in vivo transduction. $\boldsymbol{b}$ shows viral-mediated GFP immunostaining within the RVM, indicating successful viral transduction. $\boldsymbol{c}$, Double immunostaining for GFP and NP1 demonstrated that expression of NP1 shRNAi (but not random shRNAi) vector resulted in a loss of NP1 immunoreactivity in RVM sections from animals killed $2 \mathrm{~d}$ after SNI. $\boldsymbol{d}$, Quantitative analysis showed that the silencing vector reduced the number of RVM NP1-immunoreactive neurons at this time point $\left(\boldsymbol{d} ; t=2.008 ; \mathrm{df}=9\right.$; ${ }^{*} p=0.04$, one-tailed $t$ test). $\boldsymbol{e}$, Microinjection of a lentiviral vector driving NP1 overexpression ( $\mathrm{PWPI-NP1+)}$ increased RVM NP1 mRNA relative to that of the control vector (overexpression effect, $F_{(1,20)}=22.14, p=0.0001$, two-way ANOVA). NP1 shRNAi inhibited NP1 mRNA overexpression induced by the pWPI-NP1+ vector (silencing effect, $F_{(1,20)}=18.02, p=0.0004$, overexpression $x$ silencing interaction, $F_{(1,20)}=10.39, p=0.004$, two-way ANOVA). A nonsignificant decrease of basal NP1 was observed ( ${ }^{\#} p<0.05$ vs control group; ${ }^{*} p<0.05$ vs NP1+/Random shRNAi group, Bonferroni's post hoc tests). $f$, RVM infusion of NP1-shRNAi $7 d$ before SNI prevented mechanical allodynia (left, test day effect, $F_{(6,78)}=43.9, p<0.001$, test day $\times$ silencing interaction, $F_{(6,78)}=9.7, p<0.001$, two-way ANOVA) and attenuated hyperalgesia of the ipsilateral hindpaw (right panel, test day effect, $F_{(6,78)}=36.3, p<0.001$, test day $\times$ silencing interaction, $F_{(6,78)}=12.7, p<0.001,2$-way ANOVA) relative to infusion of a control vector ( ${ }^{*} p<0.05$ vs Random shRNAi, Bonferroni's post hoc tests). $\boldsymbol{g}$, RVM infusion of the silencing vector $24-48 \mathrm{~h}$ after SNI attenuates the expression of SNI-induced mechanical allodynia (left: test day effect, $F_{(4,36)}=58.3, p<0.001$; test day $\times$ silencing interaction, $F_{(4,36)}=5.2, p=0.002$, two-way ANOVA) and hyperalgesia (right: test day effect, $F_{(4,36)}=30.1, p<$ 0.001 ; test day $\times$ silencing interaction, $F_{(4,36)}=12.1, p<0.001$, two-way ANOVA). ${ }^{*} p<0.05$ vs SNI Random shRNAi, Bonferroni's post hoc tests. No effect of NP1 silencing on basal thresholds is observed in control (sham-operated) animals. $\boldsymbol{h}$, Left, NP1 K0 mice show attenuated SNI-induced mechanical allodynia (test day effect, $F_{(4,48)}=67.7, p<0.001$; test day $\times$ genotype interaction, $F_{(4,48)}=3.54, p=0.013$, two-way ANOVA; ${ }^{*} p<0.05$ vs WT SNI, Bonferroni's post hoc tests). Right, Rescuing NP1 expression selectively in the RVM of NP1-deficient mice with a lentiviral vector driving overexpression of NP1 (NP1+) restores the pain phenotype in NP1 K0 mice [all groups are NP1 K0 mice, they are infused with NP1 overexpressing (NP1+) or control (CONT) vectors before sham or SNI surgery; test day effect, $F_{(7,77)}=40.6, p<0.001$; test day $\times$ vector interaction, $F_{(7,77)}=3.60, p=0.002$, two-way ANOVA; $p<0.05$ vs CONT SNI, Bonferroni's post hoc tests]. For all panels, arrows indicate time of infusion. Number of animals per group is shown in parenthesis. Bsln, Baseline measurements. Data represent means \pm SEM.

mary neuronal cell cultures (DeGregorio-Rocasolano et al., 2001; Clayton et al., 2012) and hypoxic-ischemic injury in the neonatal brain (Hossain et al., 2004). The association of NP1 with dystrophic neurites surrounding tau deposits and amyloid plaques in brain samples from Alzheimer patients has also been described (Abad et al., 2006). Moreover, NP1 has been involved in synaptogenesis and the clustering of Glu receptors (Xu et al., 2003; Sia et al., 2007), and in synapse refinement in the developing visual system (Bjartmar et al., 2006). However, the majority of these observations derive from in vitro cell culture preparations or brains in early postnatal developmental stages. Thus, the physiological function for NP1 in vivo and in the adult brain has not been clearly established (Bjartmar et al., 2006). Our observations derived from a rodent model of chronic neuropathic pain are 
consistent with a role for NP1 in functional plasticity in the adult brain. Specifically, we have identified a role for NP1 in the plastic processes leading to and maintaining chronic neuropathic pain.

It is worth noting that NP1 immunoreactivity appears confined to the nuclear compartment under control conditions. This is consistent with the cNLS Mapper prediction score of $\alpha$-dependent nuclear localization signals in NP1 (http://nls-mapper.iab.keio.ac.jp; bipartite score: 5.7; sequence: EKGQKDNRPGDKFQLTFPLRTNYMYAKVKK) (Kosugi et al., 2009). Although NP1 has been predicted to be a secreted protein (Kirkpatrick et al., 2000), it has also been found located in intracellular compartments (Clayton et al., 2012). Activated NP1 has been shown to distribute in puncta throughout the cytoplasm and to be associated with mitochondria and involved in mitochondrial trafficking (Clayton et al., 2012). Interestingly, following nerve injury, NP1 was also seen localized to puncta distributed throughout the cytoplasm. At present, it is not apparent from our data whether this subcellular redistribution is necessary for the pain-facilitating effects of NP1 in our neuropathic pain model. However, the observation that silencing NP1 expression attenuates the SNI-induced hyperalgesia and allodynia indicates that translocation of existing protein alone is not sufficient and that newly synthesized NP1 is required for these effects.

Interestingly, basal nociception was not altered in NP1 KO mice. Similarly, inhibition of NP1 expression in the RVM of sham-operated animals did not change basal pain sensitivity. This suggests that NP1 is not involved in normal, physiological, pain transmission. Rather, it seems to be specifically recruited for and to play a role in pathological pain estates like chronic neuropathy arising from nerve injury. The ability to interfere with pathological, chronic pain states but not with normal nociception has potentially important therapeutic implications.

Consistent with the literature, allodynia and hyperalgesia were fully developed $24-48 \mathrm{~h}$ after SNI. Importantly, inhibition of NP1 48 h after SNI also attenuated nerve injury-evoked allodynia and hyperalgesia. This observation indicates that NP1 is necessary for the maintenance of neuropathic pain and that blockade of NP1 expression can reverse the ongoing neuropathy even after the nerve injury insult has already occurred.

Peripheral nerve injury induces synaptic reorganization and remodeling of brain circuitry (Navarro et al., 2007). The RVM integrates information from higher brain areas and descending projections to the spinal cord dorsal horn modulate the ascending nociceptive input (Ren and Dubner, 2002). After nerve injury, adaptations within the RVM lead to facilitation of spinal cord nociceptive output, exacerbation of primary hyperalgesia, and enhanced sensory input from adjacent regions (i.e., secondary hyperalgesia) (Burgess et al., 2002). Furthermore, recent data indicate that increased descending facilitation from the RVM is necessary for the development and maintenance of neuropathic pain (Burgess et al., 2002; Vera-Portocarrero et al., 2006) and is dependent on glutamatergic transmission (Sanoja et al., 2008). Our data confirm these studies, establishing a critical role for the RVM in the maintenance of neuropathic pain, and place NP1 as one of the elements mediating the long-term changes in RVM function that sustain chronic neuropathic pain.

Although the precise mechanisms by which NP1 mediates these effects are unknown, given the established role of NP1 in synaptic reorganization and AMPA receptor clustering (O'Brien et al., 1999; Xu et al., 2003; Sia et al., 2007), it is tempting to hypothesize that NP1 in the RVM facilitates neuropathic pain by enabling plastic changes within descending pain pathways that result in mechanical hypersensitivity, a hallmark of neuropathic pain. Based on the functional responses to noxious stimuli, RVM cells have been characterized as ON, OFF, or Neutral cells (Fields and Heinricher, 1985). In chronic pain states, allodynia and hyperalgesia are maintained by increased firing of ON cells, decreased firing of OFF cells, or both (Heinricher et al., 2009). Both the ON and OFF cell populations are GABA neurons that project to the spinal cord, while 5-HT cells are mainly Neutral (Pedersen et al., 2011). In addition, a population of ON cells includes intrinsic GABA interneurons that are able to modulate local activity. A number of scenarios are consistent with NP1-mediated increased excitability of GABA cells within the RVM resulting in SNI-induced allodynia and hyperalgesia. Those include, but are not limited to, increased activity of $\mathrm{ON}$ cells, increased excitability of intrinsic GABA neurons resulting in enhanced inhibitory tone onto OFF cells, or both.

The present study provides new insights as to the role of NP1 in vivo in the adult brain. Furthermore, they suggest that targeting NP1 may be effective for the treatment of persistent pain.

\section{References}

Abad MA, Enguita M, DeGregorio-Rocasolano N, Ferrer I, Trullas R (2006) Neuronal pentraxin 1 contributes to the neuronal damage evoked by amyloid- $\beta$ and is overexpressed in dystrophic neurites in Alzheimer's brain. J Neurosci 26:12735-12747.

Bjartmar L, Huberman AD, Ullian EM, Rentería RC, Liu X, Xu W, Prezioso J, Susman MW, Stellwagen D, Stokes CC, Cho R, Worley P, Malenka RC, Ball S, Peachey NS, Copenhagen D, Chapman B, Nakamoto M, Barres BA, Perin MS (2006) Neuronal pentraxins mediate synaptic refinement in the developing visual system. J Neurosci 26:6269-6281.

Bourquin AF, Süveges M, Pertin M, Gilliard N, Sardy S, Davison AC, Spahn DR, Decosterd I (2006) Assessment and analysis of mechanical allodynia-like behavior induced by spared nerve injury (SNI) in the mouse. Pain 122:14.e1-14.e14.

Burgess SE, Gardell LR, Ossipov MH, Malan TP Jr, Vanderah TW, Lai J, Porreca F (2002) Time-dependent descending facilitation from the rostral ventromedial medulla maintains, but does not initiate, neuropathic pain. J Neurosci 22:5129-5136.

Chaplan SR, Bach FW, Pogrel JW, Chung JM, Yaksh TL (1994) Quantitative assessment of tactile allodynia in the rat paw. J Neurosci Methods 53:55-63.

Clayton KB, Podlesniy P, Figueiro-Silva J, López-Doménech G, Benitez L, Enguita M, Abad MA, Soriano E, Trullas R (2012) NP1 regulates neuronal activity-dependent accumulation of BAX in mitochondria and mitochondrial dynamics. J Neurosci 32:1453-1466.

Decosterd I, Woolf CJ (2000) Spared nerve injury: an animal model of persistent peripheral neuropathic pain. Pain 87:149-158.

DeGregorio-Rocasolano N, Gasull T, Trullas R (2001) Overexpression of neuronal pentraxin 1 is involved in neuronal death evoked by low $\mathrm{K}(+)$ in cerebellar granule cells. J Biol Chem 276:796-803.

Fields HL, Heinricher MM (1985) Anatomy and physiology of a nociceptive modulatory system. Philos Trans R Soc Lond B Biol Sci 308:361-374.

Heinricher MM, Tavares I, Leith JL, Lumb BM (2009) Descending control of nociception: specificity, recruitment and plasticity. Brain Res Rev 60:214-225.

Hossain MA (2008) Hypoxic-ischemic injury in neonatal brain: involvement of a novel neuronal molecule in neuronal cell death and potential target for neuroprotection. Int J Dev Neurosci 26:93-101.

Hossain MA, Russell JC, O’Brien R, Laterra J (2004) Neuronal pentraxin 1: a novel mediator of hypoxic-ischemic injury in neonatal brain. J Neurosci 24:4187-4196.

Kirkpatrick LL, Matzuk MM, Dodds DC, Perin MS (2000) Biochemical interactions of the neuronal pentraxins. Neuronal pentraxin (NP) receptor binds to taipoxin and taipoxin-associated calcium-binding protein 49 via NP1 and NP2. J Biol Chem 275:17786-17792.

Kosugi S, Hasebe M, Tomita M, Yanagawa H (2009) Systematic identification of cell cycle-dependent yeast nucleocytoplasmic shuttling proteins by prediction of composite motifs. Proc Natl Acad Sci USA 106:10171-10176.

Levin S, Pearsall G, Ruderman RJ (1978) Von Frey's method of measuring pressure sensibility in the hand: an engineering analysis of the WeinsteinSemmes pressure aesthesiometer. J Hand Surg Am 3:211-216. 
Navarro X, Vivó M, Valero-Cabré A (2007) Neural plasticity after peripheral nerve injury and regeneration. Prog Neurobiol 82:163-201.

O'Brien RJ, Mammen AL, Blackshaw S, Ehlers MD, Rothstein JD, Huganir RL (1997) The development of excitatory synapses in cultured spinal neurons. J Neurosci 17:7339-7350.

O’Brien RJ, Lau LF, Huganir RL (1998) Molecular mechanisms of glutamate receptor clustering at excitatory synapses. Curr Opin Neurobiol 8:364-369.

O’Brien RJ, Xu D, Petralia RS, Steward O, Huganir RL, Worley P (1999) Synaptic clustering of AMPA receptors by the extracellular immediateearly gene product Narp. Neuron 23:309-323.

Pedersen NP, Vaughan CW, Christie MJ (2011) Opioid receptor modulation of GABAergic and serotonergic spinally projecting neurons of the rostral ventromedial medulla in mice. J Neurophysiol 106:731-740.

Ren K, Dubner R (2002) Descending modulation in persistent pain: an update. Pain 100:1-6.

Sanoja R, Vanegas H, Tortorici V (2008) Critical role of the rostral ventromedial medulla in early spinal events leading to chronic constriction injury neuropathy in rats. J Pain 9:532-542.

Schepers RJ, Mahoney JL, Gehrke BJ, Shippenberg TS (2008) Endogenous kappa-opioid receptor systems inhibit hyperalgesia associated with localized peripheral inflammation. Pain 138:423-439.

Sia GM, Béïque JC, Rumbaugh G, Cho R, Worley PF, Huganir RL (2007) Interaction of the N-terminal domain of the AMPA receptor GluR4 sub- unit with the neuronal pentraxin NP1 mediates GluR4 synaptic recruitment. Neuron 55:87-102.

Tal M, Bennett GJ (1994) Extra-territorial pain in rats with a peripheral mononeuropathy: mechano-hyperalgesia and mechano-allodynia in the territory of an uninjured nerve. Pain 57:375-382.

Tsui CC, Copeland NG, Gilbert DJ, Jenkins NA, Barnes C, Worley PF (1996) Narp, a novel member of the pentraxin family, promotes neurite outgrowth and is dynamically regulated by neuronal activity. J Neurosci $16: 2463-2478$.

Urban MO, Gebhart GF (1999) Supraspinal contributions to hyperalgesia. Proc Natl Acad Sci U S A 96:7687-7692.

Vanegas H, Schaible HG (2004) Descending control of persistent pain: inhibitory or facilitatory? Brain Res Brain Res Rev 46:295-309.

Vera-Portocarrero LP, Zhang ET, Ossipov MH, Xie JY, King T, Lai J, Porreca F (2006) Descending facilitation from the rostral ventromedial medulla maintains nerve injury-induced central sensitization. Neuroscience 140:1311-1320.

Xu D, Hopf C, Reddy R, Cho RW, Guo L, Lanahan A, Petralia RS, Wenthold RJ, O'Brien RJ, Worley P (2003) Narp and NP1 form heterocomplexes that function in developmental and activity-dependent synaptic plasticity. Neuron 39:513-528.

Zimmermann M (1983) Ethical guidelines for investigation of experimental pain in conscious animals. Pain 16:109-110. 